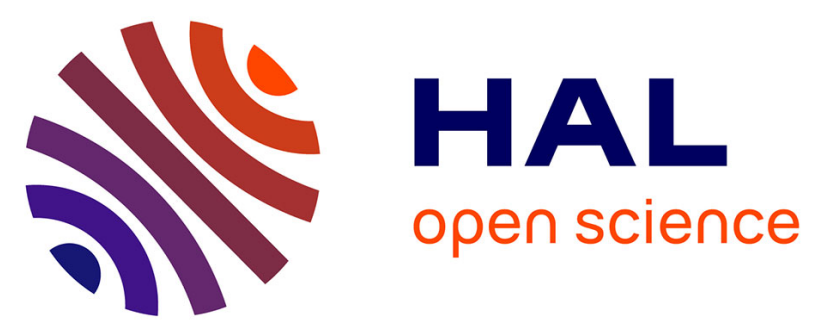

\title{
The influence of the effectors of yeast pyruvate decarboxylase (PDC) on the conformation of the dimers and tetramers and their pH-dependent equilibrium
}

S. König, D. Svergun, M. Koch, G. Hübner, A. Schellenberger

\section{- To cite this version:}

S. König, D. Svergun, M. Koch, G. Hübner, A. Schellenberger. The influence of the effectors of yeast pyruvate decarboxylase (PDC) on the conformation of the dimers and tetramers and their pH-dependent equilibrium. Journal de Physique IV Proceedings, 1994, 04 (C9), pp.C9-303-C9-307. 10.1051/jp4:1994953 . jpa-00253515

\section{HAL Id: jpa-00253515 https://hal.science/jpa-00253515}

Submitted on 1 Jan 1994

HAL is a multi-disciplinary open access archive for the deposit and dissemination of scientific research documents, whether they are published or not. The documents may come from teaching and research institutions in France or abroad, or from public or private research centers.
L'archive ouverte pluridisciplinaire HAL, est destinée au dépôt et à la diffusion de documents scientifiques de niveau recherche, publiés ou non, émanant des établissements d'enseignement et de recherche français ou étrangers, des laboratoires publics ou privés. 


\title{
The influence of the effectors of yeast pyruvate decarboxylase (PDC) on the conformation of the dimers and tetramers and their pH-dependent equilibrium
}

\author{
S. König, D. Svergun*, M.H.J. Koch*, G. Hübner and A. Schellenberger \\ Institute of Biochemistry, Department of Enzymology, Martin-Luther-University Halle-Wittenberg, \\ Weinbergweg 16a, 06099 Halle, Germany \\ * European Molecular Biology Laboratory, Hamburg Outstation, EMBL c/o DESY, Notkestr. 85, \\ 22603 Hamburg, Germany
}

\begin{abstract}
The influence of effectors of yeast pyruvate decarboxylase, phosphate, pyruvamide, thiamin diphosphate and $\mathrm{Mg}+$, on the $\mathrm{pH}-$ dependent equilibrium between dimers and tetramers was studied by synchrotron radiation $\mathrm{X}$-ray solution scattering. Thiamin diphosphate and phosphate shift the equilibrium to higher values without altering the structure of the oligomers. Pyruvamide, a substrate analogue activator, induces a significant change in the structure of the tetramer. By eliminating radiation damage by addition of dithioerythrol to the buffers, the scattering curves could be measured accurately over a large angular range. They were expanded in terms of spherical harmonics to obtain the shapes of the dimers and tetramers with higher resolution than was hitherto possible. This also allowed us to position the dimers, which are centrosymmetric at low resolution, in the tetramers which have 222 symmetry. The results indicate that addition of pyruvamide results in a less compact tetramer owing to structural changes in the dimers and to their displacements.
\end{abstract}

\section{Introduction}

Solutions of yeast pyruvate decarboxylase (PDC), an enzyme involved in one of the steps of alcoholic fermentation, are characterized by a pH-dependent equilibrium between dimers and tetramers of the $\alpha$ and $\beta$ subunits and the catalytic activity of the enzyme is related to the fractions of tetramers in the solutions [1]. In the experiments reported below, the influence of various effectors, phosphate, thiamin diphosphate and $\mathrm{Mg}^{++}$, and pyruvamide, on this equilibrium was studied. Phosphate is known to enhance cooperativity in the tetramer while reducing ist affinity for the substrate [2]. Thiamin diphosphate and $\mathrm{Mg}^{+}$are the natural cofactors of PDC and their catalytic effects have been studied in detail [3-5]. Pyruvamide can replace the substrate, pyruvate, as an activator $[6,7]$ converting the reaction rate curves from sigmoid to hyperbolic. In previous experiments, radiation damage resulted in the progressive formation of octamers, an effect which made it difficult to obtain $\mathrm{X}$-ray scattering patterns with good statistics. This difficulty was eliminated by addition of dithioerythrol to the buffers and more reliable scattering curves covering a larger range of scattering vectors were recorded. From these, shapes with higher resolution than those previously reported [1] could be obtained for the dimers and the tetramers by expansion in spherical harmonics. These allow one to place the models of the dimer in those of the tetramers and to visualize the structural change induced by pyruvamide.

\section{Results}

The extended scattering curves of the solutions containing essentially dimers ( $\mathrm{pH} \mathrm{9.2)} \mathrm{or} \mathrm{tetramers} \mathrm{(} \mathrm{pH}$ 6.5 ) are illustrated in Fig. 1. The orthogonal expansion method indicates that the scattering data from the dimer can be described by ten independent parameters and those of the tetramer and of the tetramer + PA 
by eleven independent parameters. The maximum sizes $\left(D_{\max }\right)$ of the dimer and native tetramer were found to be nearly identical, about $13 \mathrm{~nm}$, whereas the size of the tetramer + PA is approximately $10 \%$ larger. These estimates of $D_{\max }$ were further confirmed by the indirect transform program GNOMOKO, which gave the best results using $D_{\max }=13 \mathrm{~nm}$ for the dimer and native tetramer and $D_{\max }=15 \mathrm{~nm}$ for the tetramer + PA. The processed scattering curves and the corresponding distance distribution functions are shown in Fig. 1 and the integral geometrical parameters of the different oligomers are summarized in Table 1 .
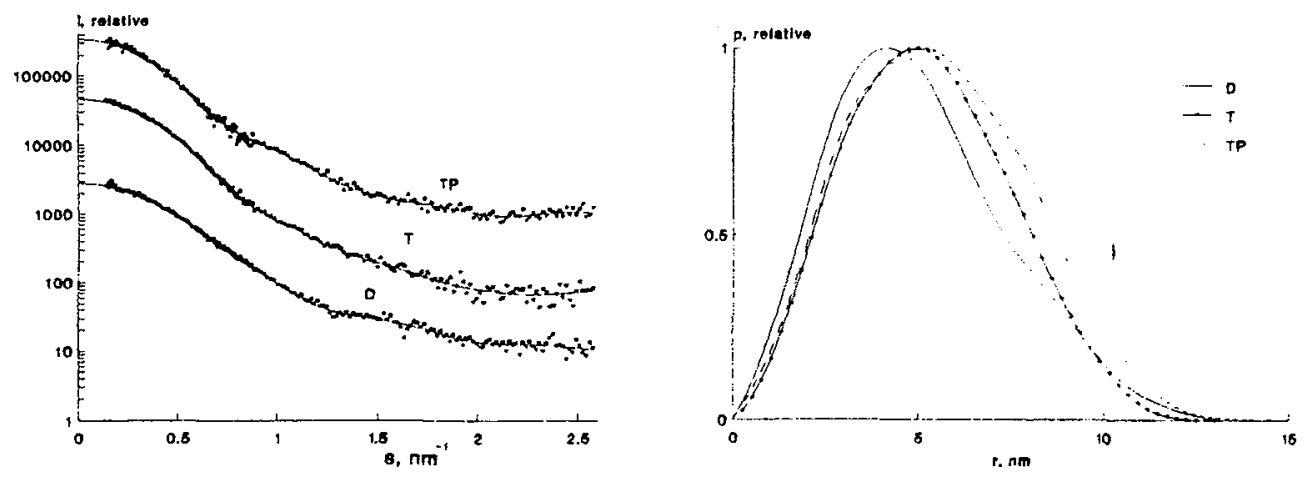

Fig. 1. Left panel: Scattering from dimers (D), tetramers (T) and tetramers with pyruvamide (TP): experimental data (dots) and fitted curves (full lines). The curves have been displaced by one logarithmic unit for better visualization. Right panel: Distance distribution functions $p(r)$ of the dimer (D), tetramer (T) and tetramer + PA (TP). The vertical bar indicates the largest propagated error.

Table 1. Geometrical parameters of dimers and tetramers and their models

\begin{tabular}{|l|c|c|c|}
\hline & $\mathrm{Rg}[\mathrm{nm}]$ & Volume $\left[\mathrm{nm}^{3}\right]$ & $D_{\max }[\mathrm{nm}]$ \\
\hline Dimer & & & \\
\hline Experimental & $3.88 \pm 0.04$ & 210 & 13.0 \\
\hline Model & 3.44 & 190 & 12.4 \\
\hline & & & \\
\hline Tetramer & & & 13.0 \\
\hline Experimental & $4.03 \pm 0.03$ & 381 & 14.1 \\
\hline Model & 4.08 & 386 & \\
\hline & & & \\
\hline $\begin{array}{l}\text { Tetramer }+ \\
\text { PA }\end{array}$ & & & 15.0 \\
\hline Experimental & $4.30 \pm 0.05$ & 381 & 14.7 \\
\hline Model & 4.28 & 380 & \\
\hline
\end{tabular}

Addition of DTE to the buffers resulted in a much higher stability of the samples in the X-ray beam as illustrated in Fig. 2 and the scattering patterns at all pH values could be fitted satisfactorily as linear combinations of those of the dimers and tetramers oniy. This also confirms that the formation of octamers in the previous measurements [1] was due to radiation damage. 


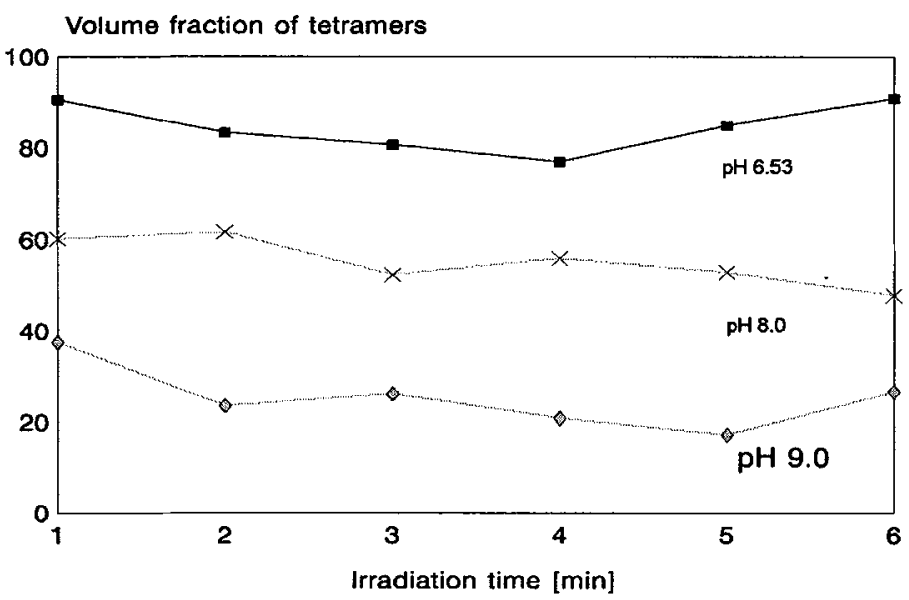

Fig. 2. Volume fractions of tetramers as a function of irradiation time for samples of pyruvate decarboxylase in buffers with different $\mathrm{pH}$ values containing $1 \mathrm{mM}$ DTE.

Oligomer content as a function of $\mathrm{pH}$ for native $\mathrm{PDC}$ and in the presence of the non-competitive inhibitor phosphate, the activator pyruvamide (PA) and the cofactors thiamin diphosphate and $\mathrm{Mg}^{++}$were calculated as described previously [1] and the results are illustrated in Fig. 3. Native PDC in the range $5.5<\mathrm{pH}<7.5$ is nearly completely tetrameric, between $\mathrm{pH} 7.5$ and 9.5 there is an equilibrium between dimers and tertamers and above pH 9.5 the solutions contain nearly exclusively dimers. The effectors increase the tetramer fraction at higher $\mathrm{pH}$-values and this effect is more pronounced with the cofactors, TDP and $\mathrm{Mg}^{++}$. The volume fraction of tetramers is still $100 \%$ at $\mathrm{pH} 8.5$ and even at $\mathrm{pH} 9.5$ it still reaches $40 \%$. The influence of phosphate and pyruvamide is less pronounced and although the onset of dissociation of the tetramers is not shifted to higher $\mathrm{pH}$-values, the volume fraction of the tetramers in the alkaline $\mathrm{pH}$-range is higher than for native PDC. It reaches about $20 \%$ with pyruvamide and $25 \%$ with phosphate. Moreover, pyruvamide alters the conformation of the tetramers as indicated by the significant increase in radius of gyration $(0.3 \mathrm{~nm})$ and the discussion below.

$\mathrm{PPC}+\mathrm{TOP} \mathrm{Mg}++$

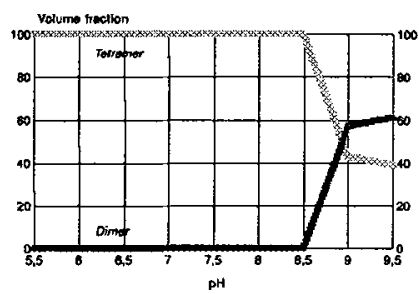

PDC + Phosphate

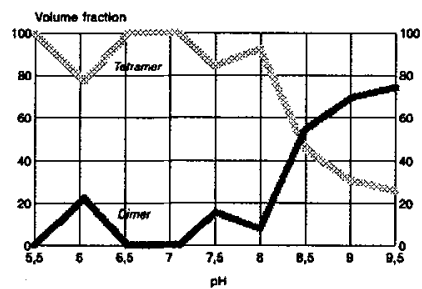

Native PDC

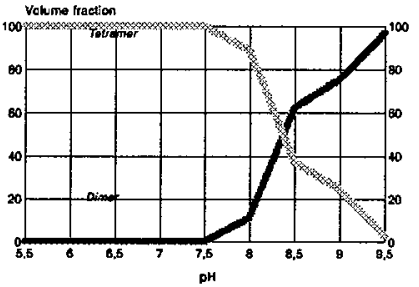

PDC + Pyruvamide

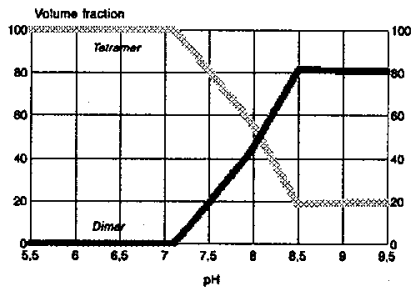

Fig. 3 Volume fractions of dimers and tetramers as a function of $\mathrm{pH}$ for native PDC and for PDC in the presence of various effectors as indicated. 
To determine the shape of the dimers and tetramers in solution it is necessary to put the experimental data on an absolute scale (i.e. the forward scattering should be equal to the square of the volume of the particles in the homogeneous approximation).

For this reason the curves were scaled using the Porod volume, a parameter which does not depend on concentration. The Porod volumes given in Table 1 were evaluated from the outer parts of the scattering patterns following standard procedures [8] and subtracting a constant to force a $s^{4}$ dependence on the intensities at larger angles. For subsequent calculations, the experimental curves for $0<s<2 \mathrm{~nm}^{-1}$ renomalized to these volumes were used. Data for $s>2 \mathrm{~nm}^{-1}$, where the contributions from inhomogeneities are significant, were discarded as they do not give additional information about the shape.

The shape of the dimer was first determined with a resolution of $L=4$ in several independent runs starting from very different initial approximations (sphere, ellipsoid, various non-centrosymmetric particles). These calculations all led to similar elongated shapes with two domains characterized by negligible contributions from the odd harmonics, $l=1$ and $l=3$ and differing only by a rotation (Fig. 4).

A

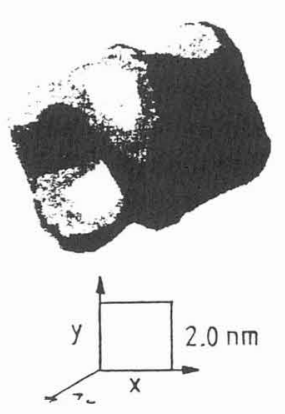

B

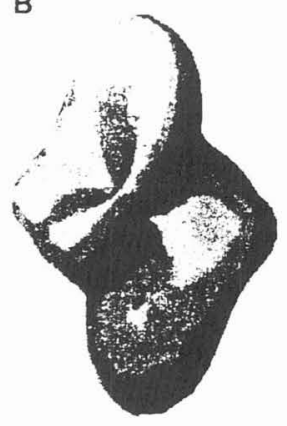

C

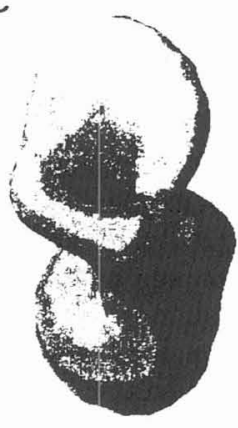

D

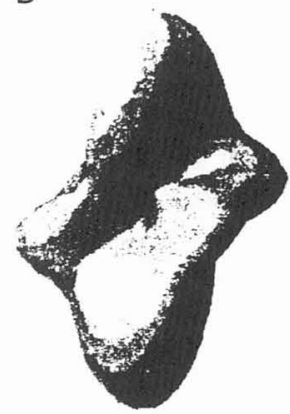

Fig. 4 A-D. Shape of dimer of $P D C$ with a resolution of $L=4$. $A$ View in the reference orientation looking down the $z$-axis. B Model A rotated by $90^{\circ}$ around the $x$-axis. C Model B rotated 45 around the $y$-axis. D Model B rotated $45^{\circ}$ around the $y$ * axis.

In the case of the tetramer, additional information can be used to achieve better resolution. Indeed, there are symmetry constraints on a stable tetramer formed by two dimers. The 222 symmetry which was previously found $[9,10]$ also exists in crystals of the $\alpha_{4}$ homotetramer [11]. Since there are no significant differences between the $\alpha$ and $\beta$ subunits at low resolution the $\alpha_{1} \beta_{2}$ tetramer should also have 222 symmetry (Fig. 5).

This imposes strong restrictions on the multipole coefficients and, if the symmetry axes coincide with the cartesian axes, all coefficients except $f_{00}, f_{20}, \operatorname{Re}\left[f_{22}\right], \operatorname{Im}\left[f_{32}\right], f_{40}, \operatorname{Re}\left[f_{42}\right], \operatorname{Re}\left[f_{44}\right] \ldots$ vanish. The shape with a resolution up to $L=6$ is then described by $13 f_{l m}$ coefficients. The number of independent parameters cannot be reduced by rotation, as the orientation is already fixed. However, at higher resolution the integral parameters of the particle (i.e. radius of gyration, volume, average intraparticle distance) which are also invariants of the scattering curve, can be fitted neatly. These invariants are expressed as combinations of the $f_{l m}$ coefficients [12] thus reducing the effective number of independent parameters. Hence, the description of the tetramer with a resolution up to $L=6$ in the 222 approximation involves again only ten independent parameters. The shapes of the tetramer and the tetramer $+\mathrm{PA}$ restored in the 222 approximation are shown in Figs. 6 and 7 together with the corresponding fits. The $R$-factors are $1.4 \%$ and $0.9 \%$, respectively. The structure parameters of the models are given in Table 1 . 


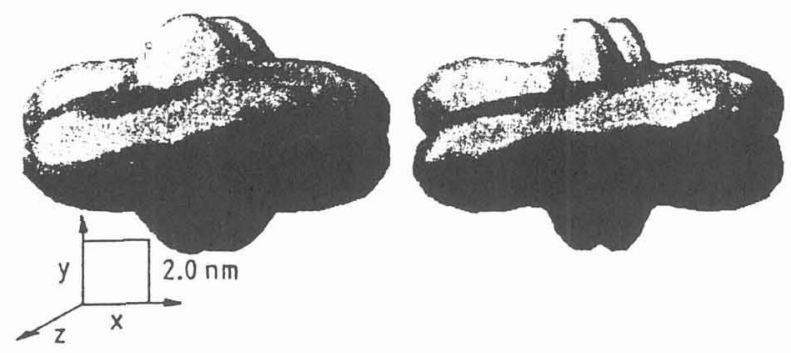

Fig. 5. Shape of the native tetramer of PDC (left) and of the tetramer + PA (right) with a resolution of $L=6$.

\section{References}

[1] König, S., Svergun, D., Koch, M. H. J., Hübner, G., Schellenberger, A. (1992) Biochem. 31, 87268731.

[2] Boitex, A., Hess, B. (1970) FEBS Lett. 9, 293-296.

[3] Schellenberger, A., Neef, H., Golbik, R., Hübner, G., König, S. (1991) Biochem. \& Physiol. of Thiamin Diphosphate Enzymes. VCH Weinheim, 3-15.

[4] Gish, G. Smyth, T. Kluger, R. (1988) J. Am. Chem. Soc. 110, 6230-6234.

[5] Zeng, X., Chung, A., Haran, M., Jordan, F. (1991) J. Am. Chem. Soc. 113, 5842-5849.

[6] Hübner, G., Schellenberger, A. (1986) Biochem. Intern. 13, 767-772.

[7] Hübner, G., Weidhase, R., Schellenberger, A. (1978) Eur. J. Biochem. 92, 175-181.

[8] Feigin, L. A., Svergun, D. I. (1987) New York, Plenum Press.

[9] Hübner, G., Schellenberger, A., Stelmaschuk, V.Y., Kiselev, N. A. (1975) Acta Biol. Med. Germ. 34, 699-701.

[10] Müller, J. J., Damaschun, G., Hübner, G.(1979) Acta Biol. Med. Germ. 38, 1-10.

[11] Dyda, F., Furey, W., Swaminnathan, S., Sax, M., Farrenkopf, B., Jordan, F. (199(1) J. Biol. Chem. 265, 17413-17415.

[12] Svergun, D.I., Stuhrmann, H. B. (1991) Acta Crystallogr. A47, 736-744. 